\title{
An infected urachal remnant
}

\author{
Yoshitaka Ueda MD, Masayuki Oki MD
}

- Cite as: CMAJ 2019 May 21;191:E562. doi: 10.1503/cmaj.181400

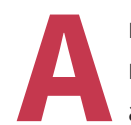

n 18-year-old man presented to the emergency department with periumbilical pain. The patient looked well and had a slight fever $\left(37.2^{\circ} \mathrm{C}\right)$. He had visited another hospital 2 years before with umbilical discharge. At that time, a subcutaneous abscess was diagnosed, which was treated by drainage and an antimicrobial agent.

In our hospital, the patient had periumbilical tenderness upon physical examination but no rebound tenderness or guarding. He had a tender erythematous nodule in the umbilicus; there was no discharge evident (Figure 1A). Ultrasonography showed a hypoechoic cystic structure that extended from the umbilicus and penetrated the abdominal muscles (Figure $1 \mathrm{C}$ ). Therefore, the lesion was unlikely to be a subcutaneous abscess but appeared to be an infected urachal remnant. Contrast-enhanced computed tomography (CT) of the abdomen identified a cystic lesion beneath the anterior abdominal wall (Figure 1B and 1D), consistent with an infected urachal remnant. After the abscess was drained, the consulting urologist prescribed oral cephalexin (1000 mg/d for $3 \mathrm{~d}$ ) and performed surgical excision of the lesion.

The urachus is an embryologic tract that extends from the bladder to the umbilicus during development of the fetus. ${ }^{1}$ Involution of the urachus is usually complete at birth, but incomplete involution results in urachal abnormalities. ${ }^{2}$ The 4 main types of urachal remnants are patent urachus or urachal fistula, umbilicalurachal sinus, vesicourachal diverticulum and urachal cyst. ${ }^{2}$

Infection is a common complication of urachal remnants and may be misdiagnosed initially, resulting in therapeutic failure and recurrence of infection., ${ }^{1,3}$ Very rarely, urachal remnants may undergo malignant transformation, so it is important that urachal remnants be differentiated from other causes of periumbilical lesions. ${ }^{4}$ Ultrasonography will differentiate urachal remnant infection from subcutaneous infection; therefore, $\mathrm{CT}$ is not required for diagnosis. ${ }^{5}$

\section{References}

1. Yu JS, Kim KW, Lee HJ, et al. Urachal remnant diseases: spectrum of CT and US findings. Radiographics 2001;21:451-61.

2. Nimmonrat A, Na-ChiangMai W, Muttarak M. Urachal abnormalities: clinical and imaging features. Singapore Med J 2008;49:930-5.

3. Schlaishunt S, Rubin J. A case of urachal remnant presenting as acute abdominal pain. J Emerg Med 1999;17:930-5.

4. Rhudd A, Moghul M, Nair G, et al. Malignant transformation of a urachal cyst a case report and literature review. J Surg Case Rep 2018;2018:rjy056.

5. Holten I, Lomas F, Mouratidis B, et al. The ultrasonic diagnosis of urachal anomalies. Australas Radiol 1996;40:2-5.

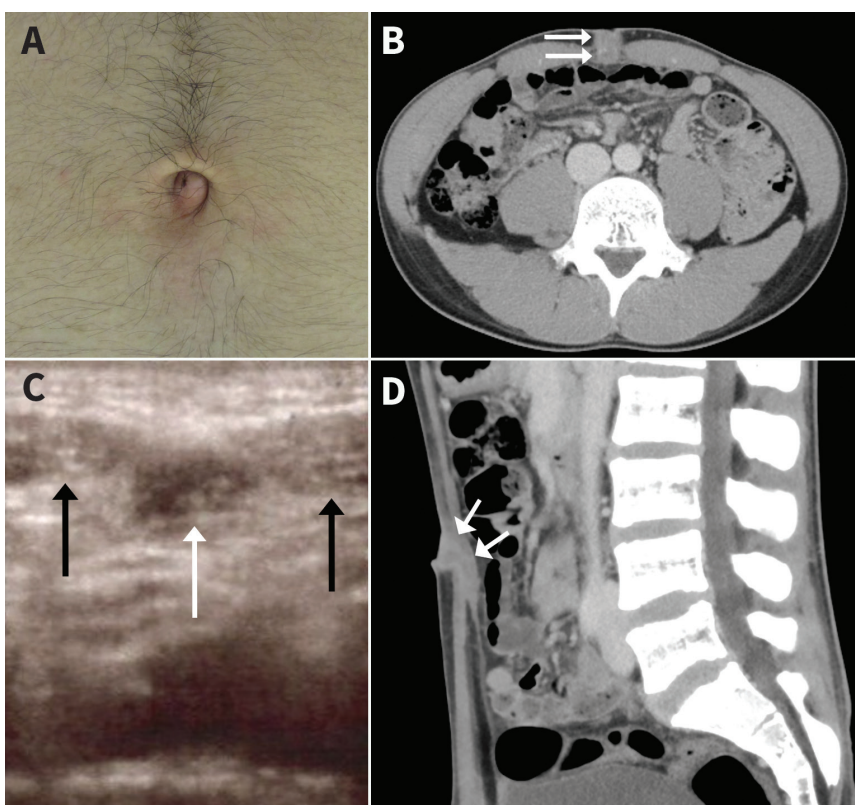

Figure 1: (A) An erythematous nodule in the umbilicus of an 18-year-old man with periumbilical pain. (B) Axial computed tomography (CT) image of the cystic lesion (white arrows) penetrating abdominal muscles, consistent with an infected urachal remnant. (C) Ultrasonography image of the lesion showing a hypoechoic cystic structure (white arrow) and abdominal muscles (black arrows). (D) Sagittal CT image of the cystic lesion (white arrows).

\section{Competing interests: None declared.}

This article has been peer reviewed.

The authors have obtained patient consent.

Affiliation: Division of General Internal Medicine, Department of Medicine, Tokai University School of Medicine, Kanagawa, Japan

Correspondence to: Masayuki Oki, okidoki@is.icc.u-tokai.ac.jp

Clinical images are chosen because they are particularly intriguing, classic or dramatic. Submissions of clear, appropriately labelled highresolution images must be accompanied by a figure caption. A brief explanation ( 300 words maximum) of the educational importance of the images with minimal references is required. The patient's written consent for publication must be obtained before submission. 